\title{
ERRATA / ERRATUM
}

No artigo "Avaliação da resposta inicial de desenvolvimento de ensaios clínicos para COVID-19 no Brasil”, DOI: 10.1590/1980-549720200104, publicado no periódico Rev bras epidemiol. 2020; 23: e200104. Na página 7, Tabela 1.

\section{Onde se lia:}

Tabela 1. Características dos protocolos de pesquisa disponíveis nas bases de dados de registro de estudos clínicos para COVID-19 em desenvolvimento no Brasil até maio 2020.

\begin{tabular}{|c|c|c|c|}
\hline \multicolumn{2}{|c|}{ Intervenções para tratamento ou profilaxia de COVID-19 (n=22) } & $\mathrm{n}$ & $(\%)$ \\
\hline \multirow{2}{*}{ Desenho do estudo } & Estudo clínico randomizado & 18 & (82) \\
\hline & Estudo clínico não randomizado & 4 & $(18)$ \\
\hline \multirow{5}{*}{ Fase da pesquisa } & Fase 1 & 3 & $(14)$ \\
\hline & Fase 2 & 5 & $(23)$ \\
\hline & Fase 3 & 8 & (36) \\
\hline & Fase 4 & 2 & (9) \\
\hline & NM & 4 & $(18)$ \\
\hline \multirow{4}{*}{ Mascaramento } & Não mascarado & 13 & (59) \\
\hline & Unicego & 1 & (5) \\
\hline & Duplo-cego & 2 & (9) \\
\hline & Quadruplo-cego & 6 & (27) \\
\hline Grupo Controle & Comparador placebo/tratamento padrão & 13 & (59) \\
\hline Multicêntrico ${ }^{a}$ & Sim & 7 & $(32)$ \\
\hline \multirow{3}{*}{ Local de recrutamento } & Hospital & 16 & $(73)$ \\
\hline & Ambulatório & 2 & (9) \\
\hline & NM & 4 & (18) \\
\hline \multirow{4}{*}{$\begin{array}{l}\text { Cálculo do Poder }^{\mathrm{b}} \\
\text { Cenário de grande tamanho de efeito } \\
\text { (RR = 0,4; incidências: } 10 \text { vs. } 25 \%)\end{array}$} & Alto $\geq 90 \%{ }^{c}$ & 1 & $(5 \%)$ \\
\hline & $\operatorname{Bom}(<90 \mathrm{e} \geq 80 \%)^{\mathrm{d}}$ & 1 & $(5 \%)$ \\
\hline & Baixo $(<80 \text { e }>50 \%)^{e}$ & 2 & $(9 \%)$ \\
\hline & Muito baixo $(<50 \%)^{\dagger}$ & 17 & $(81 \%)$ \\
\hline \multirow{4}{*}{$\begin{array}{l}\text { Cálculo do Poder } \\
\text { Cenário de moderado tamanho de efeito } \\
\text { (RR = 0,6; incidência: } 7,2 \text { vs. } 12 \% \text { ) }\end{array}$} & Alto $\geq 90 \% 9$ & 11 & $(52 \%)$ \\
\hline & $\operatorname{Bom}(<90 \text { e } \geq 80 \%)^{\mathrm{h}}$ & 1 & $(5 \%)$ \\
\hline & Baixo $(<80 \text { e }>50 \%)^{i}$ & 3 & $(14 \%)$ \\
\hline & Muito baixo $(<50 \%)^{j}$ & 6 & $(29 \%)$ \\
\hline
\end{tabular}

Intervenções para tratamento de COVID-19 $(n=21)$

\begin{tabular}{|l|c|c|c|}
\hline \multirow{3}{*}{ Critério inclusão - COVID-19 } & Diagnóstico confirmado (teste +) & 5 & $(24)$ \\
\hline & Diagnóstico suspeito - Suspeita clínica ou teste + & 12 & $(57)$ \\
\hline & Diagnóstico suspeito - Apenas suspeita clínica & 4 & $(19)$ \\
\hline \multirow{2}{*}{ Gravidade dos pacientes incluídos } & Hospitalizado moderado ou grave & 4 & $(19)$ \\
\hline & Hospitalizado grave & 5 & $(24)$ \\
\hline & Hospitalizado moderado & 1 & $(5)$ \\
\hline & Hospitalizado inespecífico & 6 & $(28)$ \\
\hline \multirow{2}{*}{$\begin{array}{l}\text { Uso de desfechos importantes para } \\
\text { COVID-19 }\end{array}$} & Não hospitalizado & 1 & $(5)$ \\
\hline
\end{tabular}

aMais que um centro de recrutamento; 'cálculo do poder não incluiu o estudo da Organização Mundial da Saúde, pelas limitações de acesso do tamanho amostral em cada braço; ${ }^{c}$ tamanho de amostra $(n)=1.986 ;{ }^{d} n=130 ;{ }^{e} n$ entre 400 e $1.000 ;{ }^{f} n$ entre 20 e $600 ;{ }^{9} n$ entre 630 e 1.968 ; ${ }^{h} n$ entre 200 e 290; ' $n$ entre 66 e 210 ; i $n$ entre 22 e 50; ${ }^{k}$ desfechos importantes conforme consenso publicado na iniciativa Core Outcome

Measures in Effectiveness Trials para pacientes hospitalizados; NM: não menciona; RR: risco relativo. 


\section{Leia-se:}

Tabela 1. Características dos protocolos de pesquisa disponíveis nas bases de dados de registro de estudos clínicos para COVID-19 em desenvolvimento no Brasil até maio 2020.

\begin{tabular}{|c|c|c|c|}
\hline \multicolumn{2}{|c|}{ Intervenções para tratamento ou profilaxia de COVID-19 ( $n=22)$} & $\mathrm{n}$ & $(\%)$ \\
\hline \multirow{2}{*}{ Desenho do estudo } & Estudo clínico randomizado & 18 & (82) \\
\hline & Estudo clínico não randomizado & 4 & (18) \\
\hline \multirow{5}{*}{ Fase da pesquisa } & Fase 1 & 3 & (14) \\
\hline & Fase 2 & 5 & (23) \\
\hline & Fase 3 & 8 & (36) \\
\hline & Fase 4 & 2 & (9) \\
\hline & NM & 4 & (18) \\
\hline \multirow{4}{*}{ Mascaramento } & Não mascarado & 13 & (59) \\
\hline & Unicego & 1 & $(5)$ \\
\hline & Duplo-cego & 2 & (9) \\
\hline & Quadruplo-cego & 6 & (27) \\
\hline Grupo Controle & Comparador placebo/tratamento padrão & 13 & (59) \\
\hline Multicêntrico ${ }^{a}$ & $\operatorname{Sim}$ & 7 & (32) \\
\hline \multirow{3}{*}{ Local de recrutamento } & Hospital & 16 & (73) \\
\hline & Ambulatório & 2 & (9) \\
\hline & NM & 4 & (18) \\
\hline \multirow{4}{*}{$\begin{array}{l}\text { Cálculo do Poder }{ }^{b} \\
\text { Cenário de moderado tamanho de efeito } \\
\text { (RR = 0,6; incidência: } 7,2 \text { vs. } 12 \%)\end{array}$} & Alto $\geq 90 \%{ }^{c}$ & 1 & $(5 \%)$ \\
\hline & $\operatorname{Bom}(<90 \text { e } \geq 80 \%)^{d}$ & 1 & $(5 \%)$ \\
\hline & Baixo $(<80 \text { e }>50 \%)^{e}$ & 2 & $(9 \%)$ \\
\hline & Muito baixo $(<50 \%)^{f}$ & 17 & $(81 \%)$ \\
\hline \multirow{4}{*}{$\begin{array}{l}\text { Cálculo do Poder }{ }^{\mathrm{b}} \\
\text { Cenário de grande tamanho de efeito } \\
\text { (RR }=0,4 \text {; incidências: } 10 \text { vs. } 25 \%)\end{array}$} & Alto $\geq 90 \%{ }^{9}$ & 10 & $(48 \%)$ \\
\hline & $\operatorname{Bom}(<90 \text { e } \geq 80 \%)^{\mathrm{h}}$ & 2 & $(9,5 \%)$ \\
\hline & Baixo $(<80 \text { e }>50 \%)^{i}$ & 2 & $(9,5 \%)$ \\
\hline & Muito baixo $(<50 \%)^{j}$ & 7 & $(33 \%)$ \\
\hline \multicolumn{4}{|c|}{ Intervenções para tratamento de COVID-19 $(n=21)$} \\
\hline \multirow{3}{*}{ Critério inclusão - COVID-19 } & Diagnóstico confirmado (teste + ) & 5 & $(24)$ \\
\hline & Diagnóstico suspeito - Suspeita clínica ou teste + & 12 & $(57)$ \\
\hline & Diagnóstico suspeito - Apenas suspeita clínica & 4 & (19) \\
\hline \multirow{6}{*}{ Gravidade dos pacientes incluídos } & Hospitalizado moderado ou grave & 4 & (19) \\
\hline & Hospitalizado grave & 5 & (24) \\
\hline & Hospitalizado moderado & 1 & (5) \\
\hline & Hospitalizado inespecífico & 6 & (28) \\
\hline & Não hospitalizado & 1 & $(5)$ \\
\hline & NM & 4 & (19) \\
\hline \multirow{2}{*}{$\begin{array}{l}\text { Uso de desfechos } \\
\text { importantes para COVID-19k }\end{array}$} & Como desfecho primário & 8 & (38) \\
\hline & Entre os desfechos secundários & 18 & (86) \\
\hline \multicolumn{4}{|c|}{ 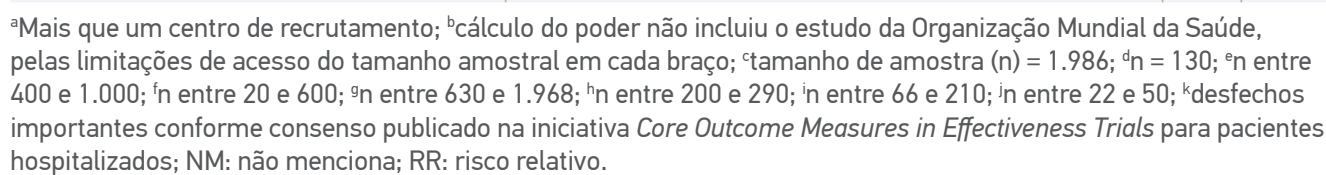 } \\
\hline
\end{tabular}

